\title{
Prevalência de depressão e Síndrome de Burnout em anestesiologistas do centro cirúrgico de hospital escola
}

\section{Prevalence of Burnout Syndrome and depression in anesthesiologists of school hospital at the surgical center}

\author{
Beatriz Cristine Hartman', Letícia Furlan², Ricardo Rasmussen Petterle³, Arlete Ana Motter ${ }^{4}$
}

${ }^{1}$ Autora para correspondência. Universidade Federal do Paraná. Matinhos, Paraná, Brasil. ORCID: 0000-0002-9408-1790. beatrizhart17@gmail.com ¿Universidade Federal do Paraná. Matinhos, Paraná, Brasil. ORCID: 0000-0002-5627-9624. fisiolefurlan@hotmail.com 3Universidade Federal do Paraná. Matinhos, Paraná, Brasil. ORCID: 0000-0001-7735-1077. estatisticoufpr@gmail.com 4Universidade Federal do Paraná. Matinhos, Paraná, Brasil. ORCID: 0000-0002-2585-207X. arlete.motter@uol.com.br

RESUMO | INTRODUÇÃO: A presença de Síndrome de Burnout (SB) e Depressão vêm aumentando devido às rotinas extenuantes vivenciadas pelos anestesiologistas. OBJETIVO: Identificar a prevalência de Depressão e Síndrome de Burnout em anestesiologistas e residentes desta especialidade médica do Centro Cirúrgico de um Hospital. MÉTODo: Estudo transversal, descritivo e exploratório. Participaram da pesquisa 51 profissionais, sendo utilizados dois questionários, ambos autoaplicáveis: os Inventários de Maslach Burnout (MBI) e Depressão de Beck, além de uma breve conversa a respeito da rotina de trabalho dos profissionais. RESULTADOS: Para a SB, verificou-se a presença desta em 3 médicos e 5 residentes. Já para a depressão; encontrou-se níveis de mínima (24 indivíduos), leve (4 indivíduos), moderada (4 indivíduos) e grave depressão (1 indivíduo). CONCLUSÃo: Embora obtido baixos valores para a SB e Depressão grave, é relevante destacar que sinais e sintomas iniciais destas patologias estão presentes em muitos profissionais, sendo que a persistência da rotina em que se encontram pode acentuar a aparição de ambas.

PALAVRAS-CHAVE: Anestesiologistas. Depressão. Burnout. Saúde do trabalhador.

\begin{abstract}
INTRODUCTION: The presence of Burnout Syndrome (BS) and Depression have been increasing, due the strenuous routines experienced by anesthesiologists. OBJECTIVE: ToidentifytheprevalenceofDepressionandBSinanesthesiologists and residents of this medical specialty of a School's Hospital. METHOD: Cross-sectional, descriptive and exploratory study. A total of 51 professionals participated in this study It was used two questionnaires, both self-applied: the Maslach Burnout Inventory (MBI) and Beck Depression Inventory, as well as a interview about the work routine of them. RESULTS: BS was detected in 3 physicians and 5 residents. Furthermore, for depression (24 individuals), mild (4 individuals), moderate (4 individuals) and severe depression (1 individual). CONCLUSION: Although low values are obtained for SB and severe depression, it is important to emphasize that initial signs and symptoms of these pathologies are present in many professionals. Besides that, if the strenuous routine persists it may accentuate or develop the symptons.
\end{abstract}

KEYWORDS: Anesthesiologists. Depression. Burnout. Occupational health. 


\section{Introdução}

O trabalho de um médico anestesiologista é caracterizado pela atuação em casos de emergência, terapia intensiva, e quadros de dor aguda e crônica, sendo responsável pela avaliação pré-anestésica do paciente, administração da anestesia e o monitoramento contínuo das suas funções vitais antes, durante e após o procedimento ao qual foi submetido 8,25 . Esta profissão é permeada por situações estressantes, que exigem decisões rápidas e um estado íntegro de atenção e prontidão $0^{3,24}$. Exige capacidade de manter-se alerta por longos períodos de relativa inatividade, reagir instantaneamente a eventos críticos, além de antecipação a eventos adversos ${ }^{22}$.

Tendo em vista que é uma classe marcada por adversidades durante seu período de trabalho, além de privações relacionadas à vida pessoal, está sujeita a diferentes fontes de estresse que podem suceder em quadros de depressão e síndrome de Burnoutt,20.

A depressão manifesta-se em um indivíduo de diferentes formas, como a mudança de humor e a perda de interesse pelas atividades diárias, além de apresentar sintomas como irritabilidade, alteração do apetite e consequente peso, distúrbios do sono, fadiga, perda da concentração e em casos mais graves, podendo culminar em suicídio ${ }^{15}$. A depressão pode ser uma das consequências do desenvolvimento da Síndrome de Burnoutt ${ }^{6,26}$.

Ao comparar a incidência de depressão entre a população em geral, anestesiologistas e residentes desta área, a porcentagem obtida é de $11 \%, 31 \%$ e $40 \%$, respectivamente. Isto decorre de um acúmulo de fatores pessoais (exigências como a precisão e pontualidade, a realização de inúmeras tarefas, dedicação excessiva ao trabalho, competitividade, contato constante com situações frustrantes); profissionais (intensa carga física e psíquica, exposição à agentes físicos, químicos e biológicos); ambientais (questões ergonômicas, ruídos, iluminação) e institucionais (hierarquia, dificuldade na interação com os demais profissionais, infraestrutura inadequada) aos quais esta classe está exposta7,24.

A síndrome de Burnout (palavra derivada do inglês que significa consumir-se) caracteriza-se pela tríade: 1) exaustão emocional, relacionada ao esgotamento físico, cansaço; 2) despersonalização, associada à falta de empatia e dificuldade em relacionar-se com pacientes e colegas de profissão; e, 3) falta de realização profissional, caracterizada pela diminuição da autoestima e da produtividade frente ao trabalho ${ }^{4,18,26}$. Esta síndrome tem se mostrado recorrente em médicos anestesiologistas devido às condições de trabalho as quais são submetidos ${ }^{4}$, sendo uma resposta física e emocional ao estresse ocupacional, afetando diretamente a qualidade de vida e o desempenho profissional dessa classe médica. Esta também está relacionada ao desencadeamento de transtornos mentais, como quadros de ansiedade e depressão $0^{20,23}$, além de distúrbios alimentares, ocasionando assim redução ou aumento de peso ${ }^{9}$.

Segundo Nanxi Zha, aproximadamente metade dos médicos vivenciam aspectos de esgotamento decorrentes do trabalho. Estudos mostram que a ocorrência de Síndrome de Burnout é significativamente maior na classe médica se comparado às demais profissões ${ }^{4,19}$, afetando assim as diferentes especialidades $^{19,21}$. Isto ocorre principalmente devido as longas jornadas de trabalho, o número limitado de profissionais, os horários rígidos, a falta de autonomia e alta rotatividade, a baixa remuneração e a dificuldade para conciliar o tempo entre trabalho, casa e família principalmente no sexo feminino. Além disso, ressalta-se o trabalho em mais de um local; as poucas horas de sono, lazer e prática de atividade física ${ }^{3,7}$.

É importante ressaltar que esse contexto de estresse ocupacional ao qual a profissão está sujeita desenvolve em médicos e residentes, quadros de fadiga física e mental. Tal fato leva a diminuição da atenção dos profissionais durante seus procedimentos, causando assim, riscos e redução na qualidade do serviço prestado aos seus pacientes, absenteísmo, acidentes de trabalho, apatia e alterações nas relações sociais ${ }^{3,21,23}$.

Ademais, destacam-se neste cenário os altos índices de suicídio e mortes violentas na classe médica, cerca de quatro vezes mais quando comparado à população em geral. Este fato ocorre principalmente pelo estresse laboral ao qual estão submetidos, associado ao desenvolvimento de transtornos psíquicos como a ansiedade, angústia e depressão ao longo da carreira. Soma-se a isso a facilidade de acesso às substâncias e as suas doses necessárias; além da maior habilidade técnica em como realizar o ato de uma forma mais eficaz ${ }^{17,23}$. 
O suicídio é resultado da soma entre saúde individual; saúde comportamental e os fatores ambientes que envolvem um indivíduo. Está relacionado não apenas ao quadro de esgotamento físico e mental, mas também a depressão e bipolaridade ${ }^{15}$ podendo ser também um dos resultados da Síndrome de Burnout ${ }^{26}$.

Outra alternativa muitas vezes encontrada por estes profissionais é a utilização excessiva de substâncias como a cafeína, tabaco, álcool, drogas, fármacos e substâncias tóxicas. Isto se torna um hábito comum entre médicos e residentes, pois tem como objetivo minimizar as tensões e possíveis frustrações laborais, além de ajudar a suportar os períodos de estresse aos quais estão subordinados ${ }^{3,25}$.

Portanto, tendo em vista o panorama geral ao qual médicos e residentes anestesiologistas estão submetidos, o objetivo do estudo foi identificar a prevalência de Síndrome de Burnout e Depressão nessa população, em um Hospital Escola de Curitiba.

\section{Metodologia}

Trata-se de um estudo transversal, descritivo e exploratório, aprovado pelo Comitê de Ética em Pesquisa do Setor de Ciências da Saúde. O objetivo foi identificar a prevalência de Síndrome de Burnout e Depressão em anestesiologistas e residentes desta especialidade médica do Centro Cirúrgico, no período de janeiro a dezembro de 2018.

Os critérios de inclusão estabelecidos para a pesquisa consistiram em: ser médico anestesiologista ou residente da especialidade do CC analisado, de ambos os gêneros, concordar voluntariamente com o estudo e assinar o Termo de Consentimento Livre e Esclarecido (TCLE), bem como trabalhar no Hospital há no mínimo 6 meses. Foram excluídos os indivíduos que não corresponderam aos critérios acima expostos ou que não estavam disponíveis para participar, devido a rotina do trabalho, nos dias e horários de coleta de dados. A seleção ocorreu conforme indicado na Figura 1.
Para a análise dos riscos à saúde mental dos participantes foram utilizados dois questionários, ambos autoaplicáveis: o Maslach Burnout Inventory (MBI) e o Inventário de Depressão de Beck.

O MBI foi utilizado para avaliar a Síndrome de Burnout. Tal instrumento foi criado por Christine Maslach, validado no Brasil por Benevides (2001), modelo utilizado por Jodas e Haddad (2009). É um dos instrumentos mais fidedignos para avaliação da SB, pois avalia diferentes subitens. $O$ questionário é composto por um total de 22 questões abordando aspectos emocionais em relação ao trabalho e a recorrência destes sintomas no dia-a-dia. Estas se dividem em 9 questões sobre exaustão emocional; 5 sobre despersonalização e 8 sobre realização profissional. Cada um destes subitens pode ser classificado em baixo, médio e alto risco, para que assim o resultado seja estabelecido ${ }^{18}$. A presença de Síndrome de Burnout pode ser determinada quando a pontuação é elevada para os itens de exaustão emocional e despersonalização e baixa para a realização profissional ${ }^{24}$.

Utilizou-se também o Inventário de Depressão de Beck, ou Beck Depression Inventory, composto por 21 itens, consistindo em uma escala de auto relato, para levantamento da intensidade dos sintomas depressivo ${ }^{11}$.

Participaram da pesquisa 34 médicos anestesiologistas do hospital analisado e 17 residentes da especialidade, com carga horária semanal de 30 ou 40 horas semanais, conforme informação disponibilizada pela gestão. A duração média da aplicação dos questionários foi de 20 a 30 minutos, inclusos neste período uma breve conversa sobre o tempo de trabalho no hospital, carga horária semanal e locais onde atuavam concomitantemente. Foram coletados também alguns dados sociodemográficos junto à administração do hospital para caracterização da amostra da pesquisa. O recrutamento foi baseado nas escalas de trabalho diárias, dependendo da disponibilidade de cada médico/residente, sendo a abordagem entre as cirurgias ou nos intervalos de descanso. Os participantes foram convidados para a pesquisa nos corredores do Centro Cirúrgico ou na Sala dos Anestesiologistas - espaço destinado para descanso exclusivo destes. 
Para o teste de correlações dos dados obtidos e a elaboração dos resultados foi utilizado o software ( $R$ Core Team, 2018) versão 3.4.4., por um estatístico da UFPR. O valor de $\mathrm{p}<0,005$ demonstra valores significativos. Foram relacionados ambos os questionários bem como cada um separadamente junto às variáveis idade, sexo, vínculo trabalhista e tempo na função.

Figura 1. Etapas da seleção dos participantes para a pesquisa

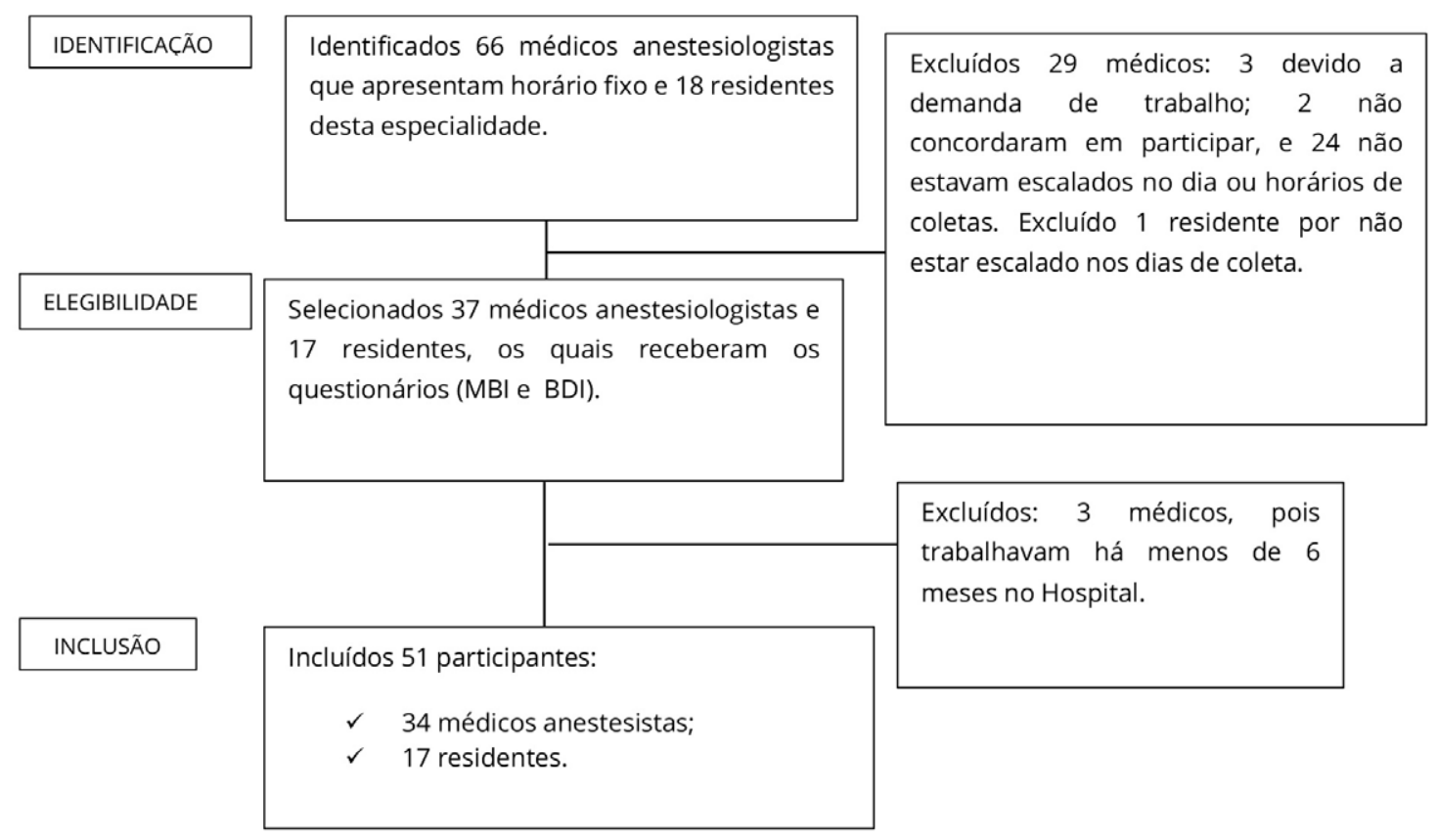

\section{Resultados e discussão}

Participaram do estudo 51 profissionais: 34 médicos anestesiologistas e 17 residentes da especialidade, sendo 22 indivíduos do sexo masculino e 29 do sexo feminino (Quadro 1). Foram identificados ao todo 66 médicos e 18 residentes no Hospital Escola, conforme informações da unidade, porém, alguns profissionais não aderiram (três participantes) à pesquisa devido à alta demanda de trabalho, a diferença de horário entre coleta e escala estabelecida ( 25 participantes) e a própria recusa em participar (dois participantes). A média de idade foi de 36 anos, abrangendo indivíduos de 25 à 66 anos; e tempo médio na função de 3 anos, variando desde 11 meses à 21 anos.

De acordo com o quadro 1, observa-se que predominam os participantes casados (16), com vínculo empregatício pelos sistema de Consolidação das Leis de Trabalho (CLT), apesar de haver outras formas de contrato de trabalho, como Cooperativa e Regime Jurídico Único (RJU). 
Quadro 1. Perfil da amostra estudada

\begin{tabular}{|c|c|}
\hline Idade & $\begin{array}{l}\text { Mínima: } 25 \text { anos } \\
\text { Máxima: } 66 \text { anos } \\
\text { Média: } 36 \text { anos } \\
\text { Desvio Padrão: 9,72 }\end{array}$ \\
\hline Sexo & $\begin{array}{l}\text { Feminino: } 29 \text { indivíduos } \\
\text { Masculino: } 22 \text { indivíduos }\end{array}$ \\
\hline $\begin{array}{l}\text { Tempo na função no hospital escola } \\
\text { estudado }\end{array}$ & $\begin{array}{l}\text { Mínimo: } 11 \text { meses } \\
\text { Máximo: } 21 \text { anos } \\
\text { Média: } 3 \text { anos }\end{array}$ \\
\hline $\begin{array}{l}\text { Vínculos empregatícios (considerando } \\
\text { apenas médicos) }\end{array}$ & $\begin{array}{l}\text { CLT: } 19 \text { indivíduos } \\
\text { COOPERATIVA: } 8 \text { indivíduos } \\
\text { RJU: } 6 \text { indivíduos }\end{array}$ \\
\hline Estado civil & $\begin{array}{l}\text { Casado: } 16 \text { indivíduos } \\
\text { Solteiro: } 13 \text { indivíduos }\end{array}$ \\
\hline
\end{tabular}

Ao considerar a prevalência da Síndrome de Burnout em profissionais da área da saúde, em especial aos de medicina, as consequências que esta pode desencadear e a população analisada obteve-se uma amostra heterogênea, conforme quadro 1. Destacam-se as escalas de trabalho de 30 ou 40 horas semanais; vínculos trabalhistas de diferentes instituições - implicando em diferença nos benefícios recebidos, regras a serem seguidas, escalas e remuneração, sendo que estes aspectos são fontes de conflitos e desconforto entre os profissionais. Além disso, é importante ressaltar que os participantes da pesquisa trabalham em outros setores do hospital analisado, bem como em outros hospitais da cidade o que aumenta a sobrecarga de trabalho.

Observou-se com o estudo maior prevalência da Síndrome de Burnout em residentes da área de anestesiologistas (cinco), e consequentemente, indivíduos com menor faixa etária, ao compará-los com os médicos (três) da mesma especialidade, corroborando com os achados na literatura ${ }^{12,13}$. Isto decorre principalmente devido a cobrança que sofrem de seus preceptores, de si mesmos e da própria sociedade. Além disso, devem cumprir jornadas extenuantes de trabalho e atividades obrigatórias ${ }^{28}$. Este fato pode estar associado também com a diferença de idade entre ambos os grupos, sendo que, na medida em que os anos passam e os profissionais adquirem maior experiência e domínio do seu trabalho, diminuem os índices da Síndrome ${ }^{1,19}$.

Tratando-se da presença de Síndrome de Burnout ao comparar o sexo masculino e feminino, obteve-se como resultado em 4 indivíduos do sexo feminino e em 4 do sexo masculino. A literatura apresenta-se ainda contraditória. Autores como Sousa (2018) e França (2012) atestam maior ocorrência no sexo feminino, devido ao nível de estresse mais elevado e ao desequilíbrio entre atividades familiares e laborais. Já Maslach, (2001), não obteve diferença significativa entre ambos, após estudo comparativo $27,16,25$.

Por fim, ao analisar o estado civil, há uma maior prevalência da SB em indivíduos solteiros, segundo a literatura, corroborando com o achado no estudo - 4 dos 5 residentes que apresentam SB são solteiros -. Ferreira et al. (2015), destaca que relacionamentos afetivos estáveis representam uma forma de "proteção" para desencadeamento da síndrome. Além disso, outro fator protetivo são os filhos. Isto é justificado devido à paternidade/maternidade desenvolver maior responsabilidade, maturidade e estabilidade ao indivíduo. 
Quadro 2. Presença de Síndrome de Burnout em médicos e residentes, conforme sexo, idade e pontuação obtida no MBI

\begin{tabular}{|c|c|c|c|c|}
\hline Médico & Idade & Exaustão Emocional & Realização & Despersonalização \\
\hline \multirow[t]{2}{*}{ Sexo feminino } & 55 anos & 54 & 25 & 18 \\
\hline & & Alto & Baixo & Alto \\
\hline \multirow[t]{2}{*}{ Sexo feminino } & 30 anos & 49 & 22 & 14 \\
\hline & & Alto & Baixo & Alto \\
\hline \multirow[t]{2}{*}{ Sexo masculino } & 30 anos & 26 & 22 & 09 \\
\hline & & Alto & Baixo & Alto \\
\hline Residente & Idade & Exaustão Emocional & Realização & Despersonalização \\
\hline \multirow[t]{2}{*}{ Sexo masculino } & 26 anos & 28 & 26 & 11 \\
\hline & & Alto & Baixo & Alto \\
\hline \multirow[t]{2}{*}{ Sexo masculino } & 29 anos & 26 & 19 & 14 \\
\hline & & Alto & Baixo & Alto \\
\hline \multirow[t]{2}{*}{ Sexo masculino } & 26 anos & 36 & 31 & 17 \\
\hline & & Alto & Baixo & Alto \\
\hline \multirow[t]{2}{*}{ Sexo feminino } & 28 anos & 26 & 32 & 15 \\
\hline & & Alto & Baixo & Alto \\
\hline \multirow[t]{2}{*}{ Sexo feminino } & 29 anos & 26 & 21 & 18 \\
\hline & & Alto & Baixo & Alto \\
\hline
\end{tabular}

O quadro 2 demonstra a presença de Síndrome de Burnout em médicos e residentes conforme sexo, idade e pontuação detalhada obtida no MBI, representando a obtenção dos resultados.

Gráfico 1. Presença de Síndrome de Burnout em médicos e residentes

\section{Síndrome de Burnout}

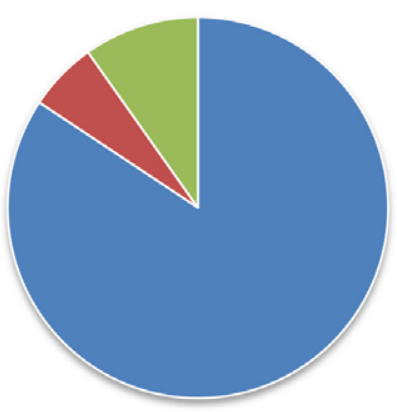

não apresenta Médicos $\quad$ Residentes 
Em relação à depressão, verificou-se apenas 1 médico com nível grave, sendo que 4 apresentaram nível moderado, 4 nível leve e 24 nível mínimo. Já em relação aos residentes, 4 destes apresentaram nível leve e 13 nível mínimo. Assim, observou-se que mesmo tendo uma mínima presença de depressão grave entre a amostra analisada, os sinais estão presentes em menores índices. Estudos mostram grande incidência de depressão ou sinais desta em médicos e residentes, sendo que este número vem aumentando, principalmente devido a tensões e cobranças do ambiente de trabalho, além de problemas pessoais. Isso afeta negativamente a segurança e o cuidado com os pacientes, culminando em possíveis erros médicos ${ }^{15,17,29}$.

O médico que apresentou nível grave de depressão também apresentou Síndrome de Burnout. Além disso, níveis moderados e leves de depressão estão associados com indivíduos que apresentaram SB, estabelecendo correlação direta entre ambas, como mostram estudos ${ }^{6,17,29}$.

Referente aos resultados encontrados para a presença de depressão em médicos anestesiologistas e residentes da especialidade, onde se utilizou o Inventário de Depressão de Beck, verificou-se níveis de mínima, leve, moderada e grave depressão, representadas no gráfico 2 e 3, respectivamente. Há diferença significativa entre os gráficos abaixo, tendo em vista que os médicos apresentam presença expressiva de depressão níveis moderado e grave, diferente do encontrado em residentes, além de um número maior de depressão grau leve.

Gráfico 2. Presença de Depressão em médicos

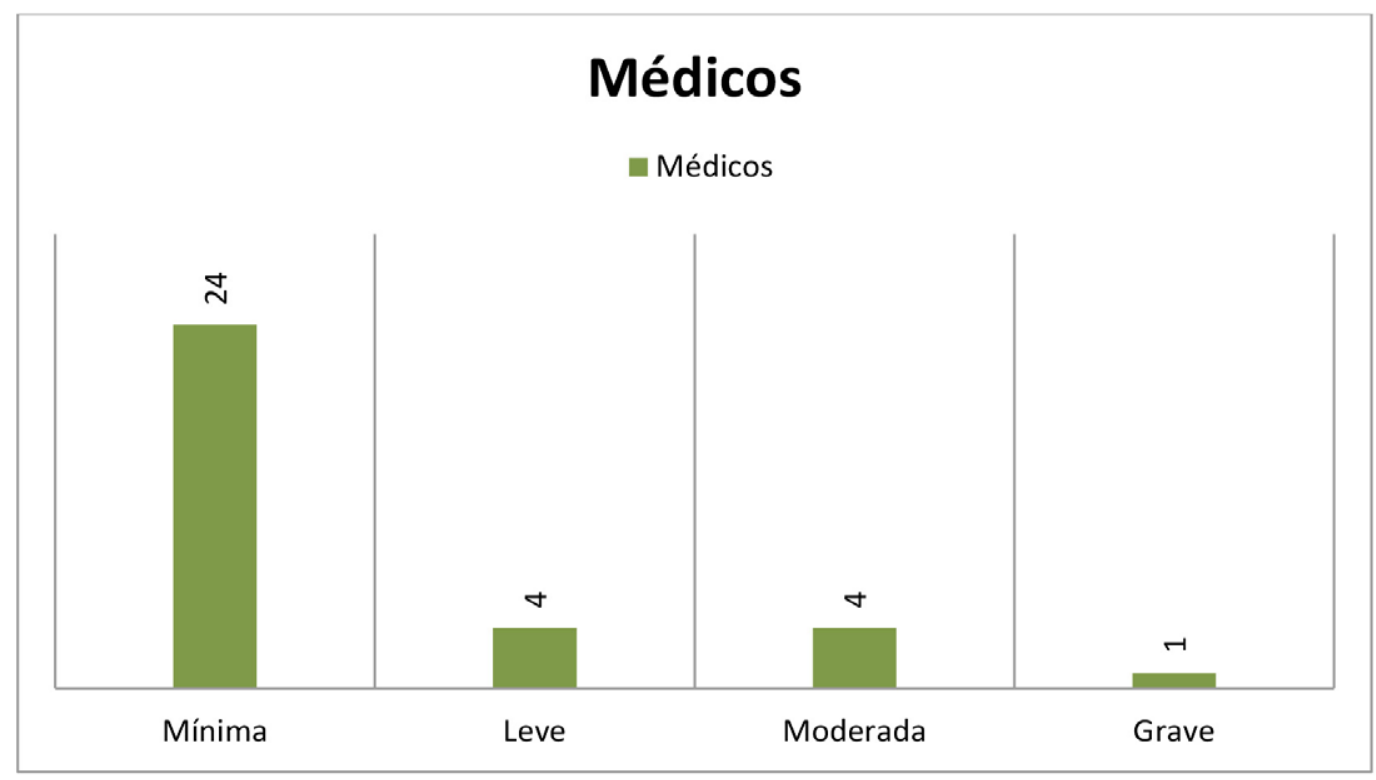

*Um dos participantes não respondeu o questionário de depressão. 


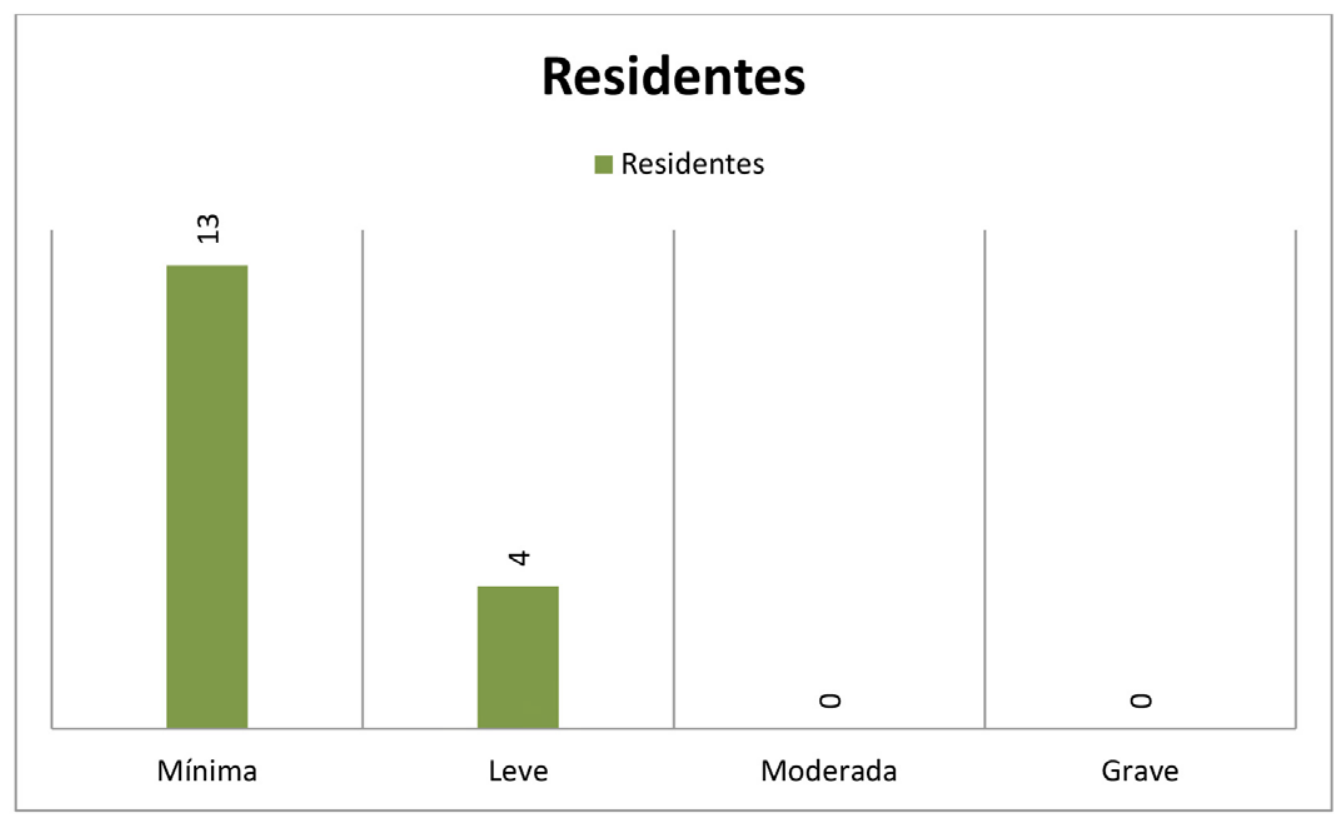

Quanto às correlações estabelecidas a partir dos dados buscados na pesquisa, como SB e idade; sexo; tempo na função; estado civil; vínculos, bem como depressão, e esta com as variáveis citadas anteriormente, obteve-se p>0,05. Isso pode ser justificado pelo $\mathrm{n}$ baixo obtido, tendo em vista a não adesão à pesquisa por parte dos profissionais, principalmente médicos, devido à rotina de trabalho, mas principalmente pelos participantes não estarem escalados nos dias ou horários da coleta de dados. Além disso, a coleta de dados realizada em apenas um dia da semana pode ter contribuído para uma amostra pequena. Quanto à jornada de trabalho semanal dos médicos e residentes, não foram estabelecidas correlações entre este aspecto e os resultados encontrados.

Dentre as principais limitações encontradas durante a realização da pesquisa, podem-se citar a pouca disponibilidade de tempo livre dos profissionais entre um procedimento e outro, pois muitas vezes precisavam arrumar os instrumentos para a próxima cirurgia, ou se deslocar para outro hospital. Pode-se acrescentar também o receio em participar da pesquisa, principalmente por considerar que poderia demorar; as interrupções constantes de outros profissionais ou a necessidade dos entrevistados saírem para acompanhar os procedimentos na sala de cirurgia. Como sugestão para novos estudos, dados como renda, carga horária semanal e uma amostra maior podem ser considerados para obtenção de novas informações.

\section{Considerações Finais}

Os dados obtidos apontam que, mesmo sendo baixo os resultados de Síndrome de Burnout e Depressão na amostra analisada, os sinais de ambas as doenças estão presentes entre os profissionais, sejam eles médicos ou residentes, decorrente não apenas do estresse ocupacional crônico, mas também das privações pessoais como períodos de sono, lazer e exercícios físicos. Estes podem não atingir a pontuação estabelecida pelos questionários, mas muitos apresentam-se próximos de desencadear a Síndrome e vem avançando nos níveis de depressão. Portanto, é fundamental que sejam revistos alguns aspectos que envolvem desde a formação destes profissionais bem como o processo de residência à atuação profissional, para que índices como esses não tenham aumentos expressivos, tendo em vista que podem afetar diretamente aspectos pessoais e profissionais do indivíduo. 


\section{Contribuições das autoras}

Hartman BC participou da concepção, coleta de dados da pesquisa, redação do artigo científico e interpretação dos dados. Furlan L participou da coleta de dados. Petterle RR participou da análise estatística dos dados da pesquisa e auxílio na interpretação dos resultados. Motter AA participou da elaboração do projeto de pesquisa e auxílio durante as coletas de dados, bem como escrita do artigo científico.

\section{Conflitos de interesses}

Nenhum conflito financeiro, legal ou político envolvendo terceiros (governo, empresas e fundações privadas, etc.) foi declarado para nenhum aspecto do trabalho submetido (incluindo, mas não se limitando a subvenções e financiamentos, participação em conselho consultivo, desenho de estudo, preparação de manuscrito, análise estatística, etc.).

\section{Referências}

1. Amoafo E, Hanbali N, Patel A, Singh P. What are the significant factors associated with burnout in doctors? Occupational Medicine. 2015;65(2):117-121. doi: 10.1093/occmed/kqu144

2. Andrade L, Gorenstein C, Vieira Filho AH, Tung TC, Artes R. Psychometric properties of the Portuguese version of the StateTrait Anxiety Inventory applied to college students: factor analysis and relation to the Beck Depression Inventory. Braz J Med Biol Res. 2001;34(3):367-374. doi: 10.1590/S0100-879X2001000300011

3. Andrade GO, Dantas RAA. Transtornos mentais e do comportamento relacionados ao trabalho em médicos anestesiologistas. Rev Bras Anestesiol. 2015;65(6):504-510. doi: 10.1016/j.bjan.2013.03.021

4. Barbosa FT, Eloi RJ, Santos LM, Leão BA, Lima FJC, SousaRodrigues CF. Correlação entre a carga horária semanal de trabalho com a síndrome de burnout entre os médicos anestesiologistas de Maceió-AL. Rev Bras Anestesiol.

2017;67(2):115-121. doi: 10.1016/j.bjan.2015.06.001

5. Benevides-Pereira AMT. MBI - Maslach Burnout Inventory e suas adaptações para o Brasil [resumo]. In: Anais da 32 Reunião Anual de Psicologia; 2001. Rio de Janeiro. 2001. p. 84-85.

6. Becker JL, Milad MP, Klock SC. Burnout, depression, and career satisfaction: Cross-sectional study of obstetrics and gynecology residentes. American J Obstet Gynecol. 2006;195(5):1444-9. doi: 10.1016/j.ajog.2006.06.075

7. Calabrese G. Impacto del estrés laboral en el anestesiólogo. Rev Col Anest. 2006;34(4): 233-240.
8. Conselho Federal de Medicina. Resolução CFM n¹.802/2006. Dispõe sobre a prática do ato anestésico. Revoga a Resolução CFM n. 1.363/1993.

9. Fang L, Hsiao LP, Fang SH, Chen BC. The associations with work stress, social support and overweight/obesity among hospital nurses: A cross-sectional study. Contemp Nurse. 2018;54(2):182194. doi: $10.1080 / 10376178.2018 .1476166$

10 Ferreira NN, Lucca SR. Síndrome de burnout em técnicos de enfermagem de um hospital público do Estado de São Paulo. Rev Bras Epidemiol. 2015;18(1):68-79. doi: 10.1590/1980$\underline{5497201500010006}$

11. Gandini RC, Martins MCF, Ribeiro MP, Santos DTG. Inventário de Depressão de Beck - BDI: validação fatorial para mulheres com câncer. Psico-USF. 2007;12(1):23-31. doi: 10.1590/S1413$\underline{82712007000100004}$

12. Govêia CS, Cruz TTM, Miranda DB, Guimarães GMN, Ladeira LCA, Tolentino FAS et al. Associação entre síndrome de burnout e ansiedade em residentes e anestesiologistas do Distrito Federal. Rev Bras Anestesiol. 2018;68(5):442-446. doi: 10.1016/j. bjan.2018.02.007

13. Hyman SA, Shotwell MS, Michaels DR, Han X, Card EB, Morse $J L$ et al. A Survey Evaluating Burnout, Health Status, Depression, Reported Alcohol and Substance Use, and Social Support of Anesthesiologists. Anesth Analg. 2017;125(6): 2009-2018. doi: 10.1213/ANE.0000000000002298

14. Jodas DA, Haddad MCL. Síndrome de Burnout em trabalhadores de enfermagem de um pronto socorro de hospital universitário. Acta paul enferm. 2009;22(2):192-7. doi: 10.1590/ S0103-21002009000200012

15. Kuhn CM, Flanagan EM. Self-care as a professional imperative: physician burnout, depression, and suicide. Can J Anesth. 2016;64(2):158-168. doi: 10.1007/s12630-016-0781-0

16. Maslach C, Schaufeli WB, Leiter MP. Job Burnout. Annu Rev Psychol. 2001;52:397-422.

17. Mata DA, Ramos MA, Bansal N, Khan R, Guille C, Di Angelantonio E et al. Prevalence of Depression and Depressive Symptoms Among Resident Physicians: A Systematic Review and Meta-analysis. JAMA. 2015;314(22):2373-2383. doi: $10.1001 /$ jama.2015.15845

18. Moreira HA, Souza KN, Yamaguchi MU. Síndrome de Burnout em médicos: uma revisão sistemática. Rev Bras Saúde Ocup. 2018;43:e3. doi: 10.1590/2317-6369000013316

19. Zha N, Patlas MN, Neuheimer N, Duszak R Jr. Prevalence of Burnout Among Canadian Radiologists and Radiology Trainees. Can Assoc Radiol J. 2018;69(4):367-372. doi: 10.1016/j. carj.2018.05.005 
20. Nyssen AS, Hansez I. Stress and burnout in anaesthesia. Curr Opin Anaesthesiol. 2008;21(3):406-411. doi: 10.1097/ ACO.0b013e3282ff85cd

21. Sanfilippo F, Noto A, Foresta G, Santonocito C, Palumbo GJ, Arcadipane A et al. Incidence and Factors Associated with Burnout in Anesthesiology: A Systematic Review. BioMed Res Int. 2017. doi: 10.1155/2017/8648925

22. Santos GM. Checklist do anestesiologista. Anestesia em revista. 2018;68(2):16-18.

23. Sociedade Brasileira de Anestesiologia. Bem-estar ocupacional em anestesiologia. Brasília: Conselho Federal de Medicina; 2013.

24. Shah A, Wyatt M, Gourneau B, Shih G, Ruyter M. Emotional exhaustion among anesthesia providers at a tertiary care center assessed using the MBI burnout survey. Psychol Health Med. 2018;24(5):620-624. doi: 10.1080/13548506.2018.1546019

25. Sousa ARC, Mourão JIB. Burnout em anestesiologia. Rev Bras Anestesiol. 2018;68(5):507-517. doi: 10.1016/j.bjane.2018.04.007

26. Kansouna Z, Boyer L, Hodgkinson M, Villes V, Lançon C, Fond G. Burnout in French physicians: a systematic review and Metaanalysis. Journal of Affective Disorders. 2019;246:132-147. doi: 10.1016/j.jad.2018.12.056

27. França FM, Ferrari R. Síndrome de Burnout e os aspectos sócio-demográficos em profissionais de enfermagem. Acta Paul Enferm. 2012;25(5):743-8. doi: 10.1590/S0103$\underline{21002012000500015}$

28. Lima FD, Buunk AP, Araújo MBJ, Chaves JGM, Muniz DLO, Queiroz LB. Síndrome de Burnout em Residentes da Universidade Federal de Uberlândia - 2004. Revista Brasileira de Educação Médica. 2007;31(2):137-146. doi: 10.1590/S010055022007000200004

29. Oliveira GS Jr, Chang R, Fitzgerald PC, Almeida MD, CastroAlves LS, Ahmad S et al. The prevalence of burnout and depression and their association with adherence to dafety and practice standards: a survey of United States anesthesiology trainees. Anesth Analg. 2013;117:182-93. doi: 10.1213/ ANE.0b013e3182917da9 